Nat. Hazards Earth Syst. Sci. Discuss., https://doi.org/10.5194/nhess-2018-92

Manuscript under review for journal Nat. Hazards Earth Syst. Sci.

Discussion started: 12 April 2018

\title{
Defining scale thresholds for geomagnetic storms through statistics
}

\author{
Judith Palacios ${ }^{1}$, Antonio Guerrero ${ }^{1}$, Consuelo Cid $^{1}$, Elena Saiz ${ }^{1}$, and Yolanda Cerrato ${ }^{1}$ \\ ${ }^{1}$ Space Weather Group, Departamento de Física y Matemáticas, Universidad de Alcalá, Alcalá de Henares, Spain. \\ Correspondence to: Judith Palacios: judith.palacios@uah.es
}

\begin{abstract}
.
Geomagnetic storms, as part of the Sun-Earth relations, are continuously monitored with different indices and scales. These indices usually have some associated scale thresholds to quantify the severity or risk of geomagnetic disturbances. However, the most usual scale thresholds are arbitrarily chosen. In this work we aim to quantify the range of the thresholds through a new method. These new thresholds are based on statistical distribution fitting.

We used different geomagnetic indices, as $D s t, S Y M-H$, and $K_{p}$, since they are relevant for space weather purposes. The first two indices have been discriminated between their negative values and the whole dataset. We considered two periods: a short-term one, comprising data from 1997 to 2012; and long-term ones, which are from 1957-2012 for Dst and 1932-2012 for $K_{p}$.

We look for the best fit for different statistical continuous distributions applied to these indices. The best fits and the data distribution functions yield to intersects that can be used to define thresholds. The best fit distribution functions are more coincidental between them when considering determined similar datasets, as non-central $f$-distribution for negative values, meaningful for geomagnetic disturbances; or non-central Student's- $t$, when the whole dataset is taken. The method yields different values for thresholds depending on the index. Thresholds for geomagnetic storms can be chosen by common values of SYM- $H$ and Dst, as $-75 \mathrm{nT}$ for moderate storms; $-150 \mathrm{nT}$ for intense storms, and $-330 \mathrm{nT}$ for extreme storms. For the case of $K_{p}$, the value equal to 5 may mark the departure from quiet time to stormy time.

The obtained values are close to those usually considered as thresholds for, typically, Dst and $K_{p}$; therefore the thresholds defined here may provide criteria to assess the vulnerability to geomagnetic activity on design or mitigation purposes.
\end{abstract}

\section{Introduction}

'Solar storms', as an example of natural hazards, is a rough term which comprises unspecified cases of eruptive and energetic solar events, such as coronal mass ejections (CMEs), flares, and also events of accelerated particles. The Sun as an active star due to its magnetic field, generates these eruptive instances - along with the solar wind-, and when CMEs are Earth directed, impinges our planet's shield, that is the Earth's magnetic field. The large-scale perturbation on the Earth's environment, named generally geomagnetic storm (or more specifically, geomagnetic disturbance) can be typically observed at mid-low latitude as a decrease of the Earth's magnetic field. Geomagnetic storms and other geomagnetic disturbances, as sudden impulses or $\mathrm{H}$-spikes, are typically measured with magnetometers in geomagnetic observatories spread across the globe. 
Nat. Hazards Earth Syst. Sci. Discuss., https://doi.org/10.5194/nhess-2018-92

Manuscript under review for journal Nat. Hazards Earth Syst. Sci.

Discussion started: 12 April 2018

(c) Author(s) 2018. CC BY 4.0 License.

Quantifying geomagnetic storm disturbances is one of the main aims in space weather. Extreme space weather events are treated now as natural environmental hazards since they pose a serious threat to different technologies, including critical infrastructures. The threats include power grid upsets, that can be damaged due to sudden changes in the Earth's magnetic field, leading to geomagnetically induced currents (GICs); problems in GNSS positioning; orbit displacement of satellites, or radio blackouts, among other undesirable effects. A summary of these risks and their economic and societal impact appeared recently in Eastwood et al. (2017). Very recently, macro-economic losses have been modelled considering worst-case scenarios centered in different areas of the globe (Schulte in den Bäumen et al., 2014).

Different geomagnetic indices have been created to account for different geophysical phenomena, and then, a scale was defined accordingly. Scales may rely on the physics behind an index, or a scale may be effect-based only. Some examples of this kind of scales are those for earthquakes - as the effect-based Mercalli scale; or as the $M_{w}$ scale, which is physics-based; for nuclear risks, the INES scale is an effect-based, and the NAMS scale is more physical (Wheatley et al., 2017). Below, we comment the most commonly employed indices for space weather.

The $D s t$ is a geomagnetic index, considered to provide a quantitative measurement of geomagnetic disturbances at low latitudes. The method for the elaboration of the Dst index is described by Sugiura and Kamei (1991), and it is assumed as a representation of the ring current enhancement encircling the Earth. This index is widely used in space weather. However, the Dst index has been questioned to misrepresent the ring current, not accounting for asymmetries (Grafe, 1998); or being inaccurate due to important seasonal offsets (Karinen and Mursula, 2006), that may even lead to a mismatch in the currently used scale. Recently, Dst is computed with 1-min resolution at https://geomag.usgs.gov/plots/dst.php. Other high-resolution (1-min) indices are widely used, as $S Y M-H$, but that index is not computed on real time.

In Gonzalez et al. (1994), the intensity of a storm was established by the minimum Dst value, and the scale thresholds of moderate and intense storm were also set. The definition for moderate storm comes from a threshold placed at $D s t$ percentile 8; and the intense storm definition comes from the Dst percentile 1, employing a dataset of 10 years for these thresholds. The superintense threshold consideration as the value $D s t<=-250 \mathrm{nT}$ (Tsurutani et al., 1992, and references therein) and employed after by e.g., Echer et al. (2008a, b) comes from a case-study ad-hoc threshold. Other arbitrary threshold of $D s t<=-400 \mathrm{nT}$ appears in Gonzalez et al. (2011). These values are tabulated in Table 1 among other indices thresholds, listed below.

The $K$ and $A$ indices (Bartels et al., 1939) have been used for decades to convey the intensity of major geomagnetic disturbances. These $K$ indices range from 0 to 9 and it is based on the maximum range of the magnetic field variation in a quasi-logarithmic scale, for any given observatory (local). The $A$ indices are derived from linearization of $K$ indices. The planetary $K_{p}$ index is an average of the $K$ index from selected ground-based magnetometers with global coverage (Bartels and Veldkamp, 1949). Similarly, its linearized version is called $A_{p}$. The indices $A_{p}$ and $K_{p}$ can be found in $3 \mathrm{~h}$ (lowercase) and $24 \mathrm{~h}$ (uppercase) resolution, e.g, $a_{p}$ is the planetary 3-h resolution description. A number of limitations of these indices is described in Rostoker (1972), such as the arbitrary upper limit of $A$, or the diurnal variation that exhibits a seasonal component for $K$.

In 1999, the U.S. National Oceanic and Atmospheric Administration (NOAA) issued a five-level geomagnetic storm scale named $G$ scale (http://www.swpc.noaa.gov/noaa-scales-explanation) that classifies geomagnetic disturbances with an effect- 
Nat. Hazards Earth Syst. Sci. Discuss., https://doi.org/10.5194/nhess-2018-92

Manuscript under review for journal Nat. Hazards Earth Syst. Sci.

Discussion started: 12 April 2018

(c) Author(s) 2018. CC BY 4.0 License.

Table 1. This table shows the thresholds of geomagnetic activity for different indices

\begin{tabular}{ccccc}
\hline Index & Quiet-minor & Moderate storm & Intense storm & Superintense \\
\hline Dst $[\mathrm{nT}]$ & $>-50$ & $-50--100$ & $<-100$ & $<-250$ \\
$K_{p}$ & $0-4$ & 5 & $7-9$ & \\
$A_{p}[2 \mathrm{nT}]$ & $0-20$ & $30-50$ & $100-400$ & \\
\hline
\end{tabular}

based scale intended on physical infrastructures, mainly for power systems. This effect-based scale stems from one of the two indices already used to measure the severity of these disturbances, which is the $K_{p}$ (Bartels and Veldkamp, 1949). $G$ is scaled from 1 to 5 (related to $K_{p}=5$ for $G 1, K_{p}=6$ for $G 2, K_{p}=7$ for $G 3, K_{p}=8-9^{-}$for $G 4$ and $K_{p}=9$ for $G 5$ ).

For more information about these geomagnetic indices, one can refer to Bartels et al. (1939); Rostoker (1972); Mayaud 5 (1980).

All this variety may pose an additional complication for the user to differentiate between physical and effect-based scales, their thresholds and their applicability range. In the end, proper threshold definition is necessary for considerations on the importance of geomagnetic disturbances and for further utilization by users and decision makers.

Some of these global geomagnetic indices are not fine tuned for the current space weather requirements, such as high temporal cadence. In addition to that, caution is recommended due to the unevenly spanned observatories around the globe (Rostoker, 1972; Karinen and Mursula, 2006). Other of the main drawbacks of most of these geomagnetic indices is that they are averages of measurements from a network of different geomagnetic observatories. This fact may provoke averaging out of important local geomagnetic disturbances and therefore, fundamental information may be missing. Rather than the consideration of a geomagnetic storm quantification as global, it is proved that very local effects may happen, such as H-spikes or Carrington-like disturbances (Cid et al., 2015; Saiz et al., 2016). The consequences of local disturbances are of the maximum relevance, since these large peaks are linked to large time derivative values, and therefore to extreme geoelectric fields, which are related to localized GIC events (Ngwira et al., 2015, and references therein).

Thresholds are not only employed to classify the severity of a natural hazard but also employed for computing the waiting time and time recurrence of events. The thresholds used for several types of natural hazards such as earthquakes, volcanic eruptions or floods are based on 1 in 50- or 1 in 100-year extremes; this approach for space weather is also applicable, as shown in different works, e.g., Love (2012); Nikitina et al. (2016); Love et al. (2016); Wintoft et al. (2016), among others.

In this paper, we use different distribution functions seeking the best fit for a number of geomagnetic indices, such as $D s t$, SYM- $H, K_{p}$; then, we define thresholds for these indices. Fitting distributions aiming at recurrence or waiting times estimations is out of the scope of this work. Opposite to the vast majority of the mentioned references, we do not use thresholds to help fitting a part or the whole distribution; threshold rationale and distribution properties are the main purposes. Hence, one of the key goals in this paper is aiming at overcoming some of the arbitrariness to establish thresholds for geomagnetic disturbances through the objective method explained in this paper. Its application can be extended to any other global or regional index. 
Nat. Hazards Earth Syst. Sci. Discuss., https://doi.org/10.5194/nhess-2018-92

Manuscript under review for journal Nat. Hazards Earth Syst. Sci.

Discussion started: 12 April 2018

(c) Author(s) 2018. CC BY 4.0 License.
Natural Hazards

and Earth System

Sciences

Discussions

(c) (i)

The paper is structured as follows: data used are presented in Section $\$ 2$, along with the preliminary statistical considerations; next in Section $\S 3$, the method is described, obtaining the best fit distributions to the indices in Section $\S 3.1$; and then in Section $\S 3.2$, the thresholds for these indices are defined by these statistical best distribution intersects. The results and discussion are presented in Section $§ 4$, and Conclusions appear in Section $§ 5$.

\section{Geomagnetic data}

In this work we made use of the following indices, which are $K_{p}, S Y M-H$ and Dst. Length duration spans to a common short period, namely from 1997 to 2012. The upper limit, 2012, is the last common year of all these indices at the time of the analyses performed, since definitive data are considered, (because data from different observatories which contribute to those indices may not be definitive when they are obtained on very recent dates). These data correspond to solar cycles 23 and 24 along with their extreme geomagnetic storms, therefore comprising two rising phases and one solar maximum. Longer periods are also considered for the analysis, which comprises the full duration of each index since its creation for historical indices (namely Dst and $K_{p}$ ). This means the period from 1932 to 2012 for $K_{p}$; and the period from 1957 to 2012 is considered for Dst. The short intervals are intended for cross comparison between all the indices, and the comparison between long and short series.

Datasets description is as follows: Dst is hourly data, with positive and negative values, with $1 \mathrm{nT}$ precision. $S Y M-H$ is 1-minute resolution data, with positive and negative values, with $1 \mathrm{nT}$ precision. The index $K_{p}$ is a special case - with $3 \mathrm{~h}$ temporal resolution - since it is scaled from 0 to 9 , and categorized with,+- and $o$, they have been assigned the corresponding values, as $+1 / 3,-1 / 3$ and .0 .

For SYM-H and Dst, we have also considered the sample of their negative values, named here as $\mid n e g($ index $) \mid$, as representative of the typical depression displayed on the geomagnetic field during the development of geomagnetic disturbances. On geomagnetic storms, negative values actually define the storm since they mean a horizontal component depression of the geomagnetic field due to a ring current enhancement. The whole distribution shape is also very relevant because all of them are quite skewed, and they are not symmetric on the positive and negative values. Since the goal of this paper is not the storm recurrence or waiting time, not timing between peak values is considered. We have compared the short and long periods in the case of historical indices.

\section{Distribution fitting and threshold definition}

\subsection{Statistical distribution fitting}

A statistical approach is used in this Section, to find the most appropriate distribution for these meaningful defined indices $|n e g(D s t)|,|n e g(S Y M-H)|, K_{p}$ and the whole Dst and SYM-H, for both short period (1997-2012) and larger periods, if applicable. We use the Python stat package, looking for the most appropriate distribution fit. We tested 82 continuous functions implemented in Python (the whole list includes 94: https://docs.scipy.org/doc/scipy-0.18.1/reference/stats.html). Most of these functions can be found, e.g, in Wilks (2011). The not used distributions due to convergence lack are the follow- 
Nat. Hazards Earth Syst. Sci. Discuss., https://doi.org/10.5194/nhess-2018-92

Manuscript under review for journal Nat. Hazards Earth Syst. Sci.

Discussion started: 12 April 2018

(c) Author(s) 2018. CC BY 4.0 License.

(c) $\underset{\mathrm{BY}}{(i)}$

Table 2. $D$ values for best fitting distributions for $S Y M-H$

\begin{tabular}{cccc}
\hline SYM-H 1997-2012 & & & \\
\hline$D$ for $|\operatorname{neg}(S Y M-H)|$ & corresponding distribution & $D$ for $S Y M-H$ & corresponding distribution \\
\hline 0.032 & non-central $f$ & 0.018 & non-central $t$ \\
0.043 & lognorm & 0.019 & Johnson-Su \\
0.043 & expon & 0.032 & genlogistic \\
0.043 & genexpon & 0.056 & $t$ \\
0.044 & Johnson-Su & 0.062 & dgamma \\
0.044 & invgamma & 0.067 & logistic \\
0.044 & invgauss & 0.067 & hypersecant \\
0.045 & genextreme & 0.069 & Frechet L (Weibull max) \\
0.045 & invweibull & 0.074 & Laplace \\
0.045 & fatiguelife & & \\
\hline
\end{tabular}

ing: arcsine,burr12, exponnorm, gausshyper, gennorm, halfgennorm, invnorm, kappa4, kappa3, levy_stable, skewnorm, trapz, vonmises_line.

These fits and the subsequent intersection points between significant distributions are utilized to define the threshold for intense, very intense and extreme cases. Note that no previous threshold to avoid the distribution bulky part is utilized to fit the functions. Sometimes the distribution bulk is not regarded for fitting distribution functions; however we consider all parts for fitting.

The minimization has been done through the Kolmogorov-Smirnoff (KS) distance parameter $D$ computation. The minimization is parametric, through the maximum likelihood estimator (MLE) used to perform the distribution fitting as default; as a double check, we also inspected the minimization by the Kolmogorov-Smirnoff method, producing the same results. Consequently, the statistics results are robust. Moreover, the fitting is not binning dependent.

Looking for the best fit, we seek the minimum value of the Kolmogorov-Smirnoff distance $D$ for all the distributions for $\mid n e g($ index $) \mid$ and the whole distribution indices. Trying to improve the histogram plots, we used the complementary cumulative distribution function (CCDF) for each index. Distribution function plotting is made by the survival function computation. The CCDF is appropriate to be fit since it avoids bin dispersion, obtaining an appropriate binning at the distribution tail (as in Riley, 2012). Binsizes vary from $5 \mathrm{nT}$ in the case of $D s t$ and $S Y M-H$ and $1 / 3$ in the case of $K_{p}$.

We list in Tables the best fits for all indices: SYM- $H$ is listed in Table 2 for the period 1997-2012; the Dst interval 1997-2012 appears in Table 3, and the period 1957-2012 is in Table 4. The $K_{p}$ intervals 1932-2012 and 1997-2012 are shown in Table 5. We have listed a maximum of 10 best distribution functions per index. In general, all $D$ are in the order of $10^{-2}$. 
Nat. Hazards Earth Syst. Sci. Discuss., https://doi.org/10.5194/nhess-2018-92

Manuscript under review for journal Nat. Hazards Earth Syst. Sci.

Discussion started: 12 April 2018

(c) Author(s) 2018. CC BY 4.0 License.

(c) (i)

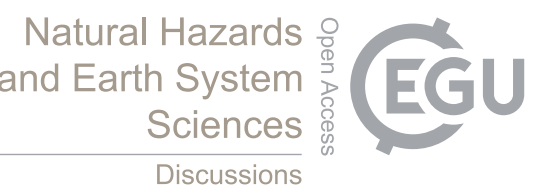

Table 3. $D$ values for best fitting distributions for $D s t$

\begin{tabular}{rccc}
\hline Dst 1997-2012 & & & \\
\hline$D$ for $|n e g(D s t)|$ & corresponding distribution & $D$ for $D s t$ & corresponding distribution \\
\hline 0.031 & non-central $f$ & 0.016 & non-central $t$ \\
0.034 & genexpon & 0.018 & Johnson-Su \\
0.041 & lognorm & 0.026 & genlogistic \\
0.042 & Johnson-Su & 0.055 & loggamma \\
0.042 & invgamma & 0.057 & Gumbel L \\
0.042 & Johnson-Sb & 0.058 & -distrib \\
0.042 & invgauss & 0.061 & powernorm \\
0.042 & powerlognorm & & \\
0.043 & non-central $t$ & & \\
0.043 & genextreme & & \\
\hline
\end{tabular}

Table 4. $D$ values for best fitting distributions for $D s t$

\begin{tabular}{rccc}
\hline Dst 1957-2012 & & & \\
\hline$D$ for $|n e g(D s t)|$ & corresponding distribution & $D$ for $D s t$ & corresponding distribution \\
\hline 0.026 & non-central $f$ & 0.013 & non-central $t$ \\
0.034 & genexpon & 0.016 & Johnson-Su \\
0.037 & powerlognorm & 0.020 & genlogistic \\
0.038 & lognorm & 0.045 & loggamma \\
0.039 & Johnson-Sb & 0.046 & Gumbel L \\
0.039 & Johnson-Su & 0.053 & powernorm \\
0.039 & invgauss & & \\
0.040 & fatiguelife & & \\
0.040 & invgamma & & \\
0.041 & non-central $t$ & & \\
\hline
\end{tabular}

\subsection{Defining thresholds through distribution best fits}

The best fits of the distribution functions to each index are clearly shown in their corresponding Figures (Figs. 1, 2, and 3). For all distributions, we can see that some parts of them have excellent fits, and some others whose fitting is very good on the bulk but not that much in the distribution tails. Then, taking advantage of this fact, a way of defining the threshold is the 5 intersects of the index CCDF with the best fit distribution, or even the intersect of the two best fit distributions. Then, we can 
Nat. Hazards Earth Syst. Sci. Discuss., https://doi.org/10.5194/nhess-2018-92

Manuscript under review for journal Nat. Hazards Earth Syst. Sci.

Discussion started: 12 April 2018

(c) Author(s) 2018. CC BY 4.0 License.

(c) (1)
Natural Hazards

and Earth System

Sciences

Discussions

Table 5. $D$ values for best fitting distributions for $K_{p}$

\begin{tabular}{|c|c|c|c|}
\hline$K_{p} 1997-2012 K_{p} 1932-2012$ & & & \\
\hline$D$ for $K_{p} 1997-2012$ & corresponding distribution & $D$ for $K_{p} 1932-2012$ & corresponding distribution \\
\hline 0.092 & invgauss & 0.070 & foldnorm \\
\hline 0.092 & Johnson-Sb & 0.070 & Gompertz \\
\hline 0.092 & lognorm & 0.075 & $f$-distrib \\
\hline 0.093 & Johnson-Su & 0.076 & Johnson-Su \\
\hline 0.096 & invgamma & 0.076 & lognorm \\
\hline 0.096 & invweibull & 0.077 & invgamma \\
\hline 0.096 & genextreme & 0.077 & Johnson-Sb \\
\hline 0.096 & fatiguelife & 0.078 & invweibull \\
\hline 0.097 & alpha & 0.078 & genextreme \\
\hline 0.097 & powerlognorm & 0.078 & invgauss \\
\hline
\end{tabular}
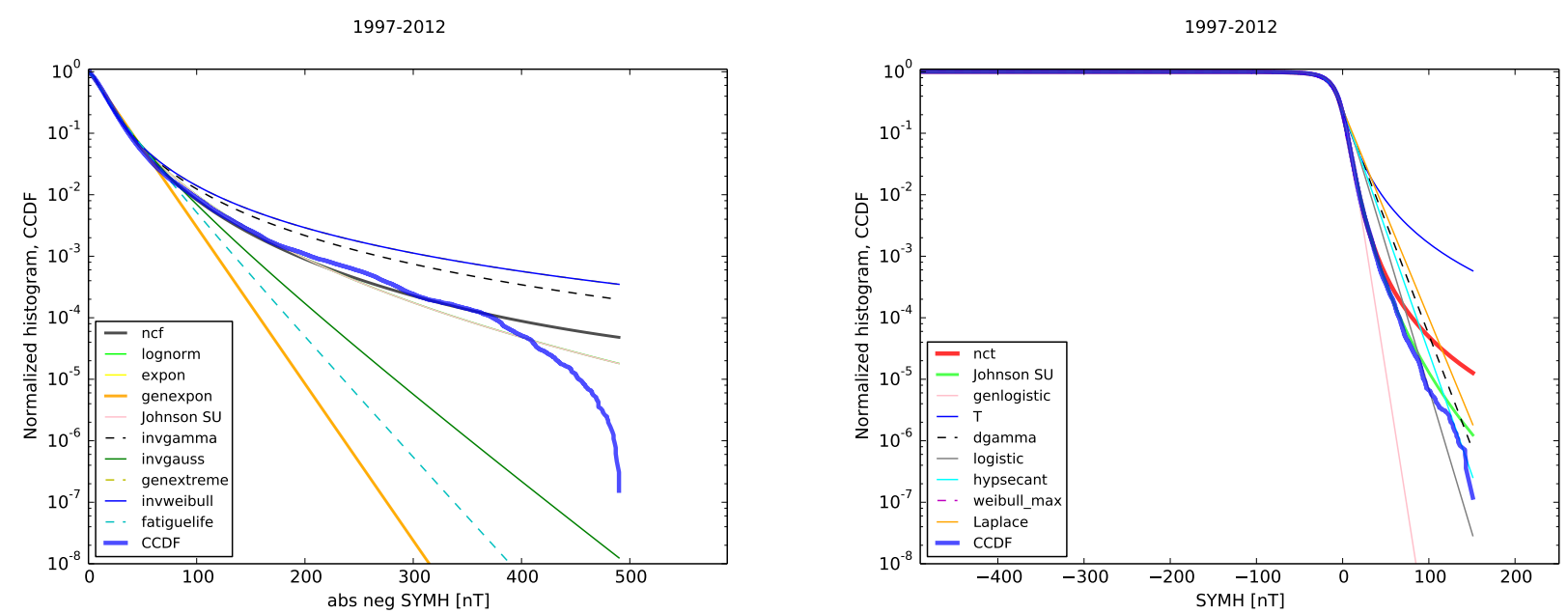

Figure 1. Best fit for $S Y M-H$ different distributions. Left: Best fit distributions for $|n e g(S Y M-H)|$, 1997-2012. Right: Best fit distributions for $S Y M-H, 1997-2012$.

define a threshold of a determined degree of geomagnetic disturbance when the intersect is in the bulk, the tail or the extreme tail. In the next Figures, we plot the CCDF with a thick blue line.

In the case of the negative values of $S Y M-H$ for the period 1997-2012, the CCDF and the best fitted functions are plotted in the left panel of Fig. 1. The CCDF starts departing from the inverse gamma ('invgamma', dashed grey) and inverse Weibull

5 (invweibull, blue thin line), at about $-50 \mathrm{nT}$. The CCDF starts the separation of the rest of functions at $-70 \mathrm{nT}$. The first depart from the non-central $f$-distribution ('ncf', which is the best fit, plotted in dark grey thick line) and Johnson-S Unbounded 
Nat. Hazards Earth Syst. Sci. Discuss., https://doi.org/10.5194/nhess-2018-92

Manuscript under review for journal Nat. Hazards Earth Syst. Sci.

Discussion started: 12 April 2018

(c) Author(s) 2018. CC BY 4.0 License.

\section{(c) (1)}

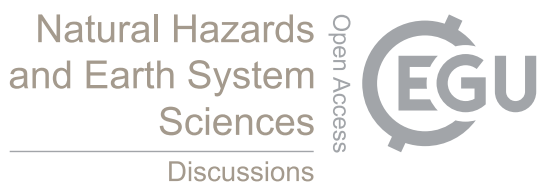

$1997-2012$

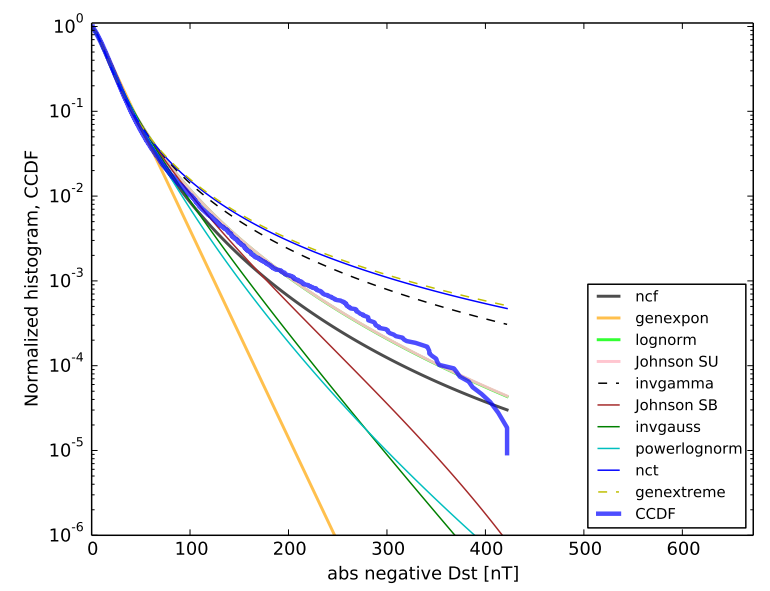

$1957-2012$

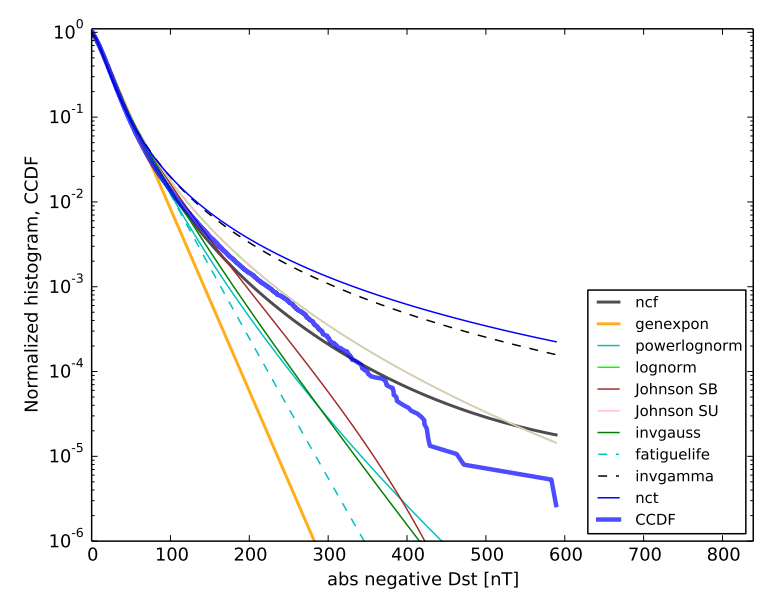

$1997-2012$

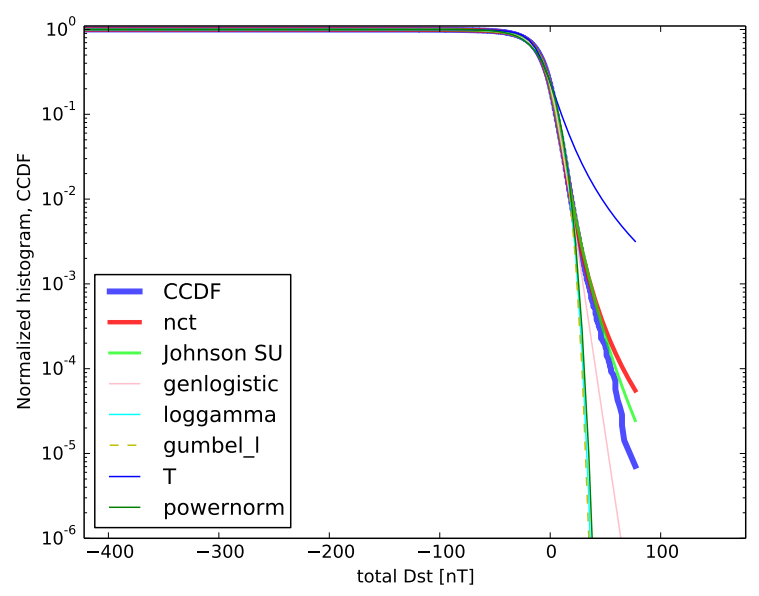

$1957-2012$

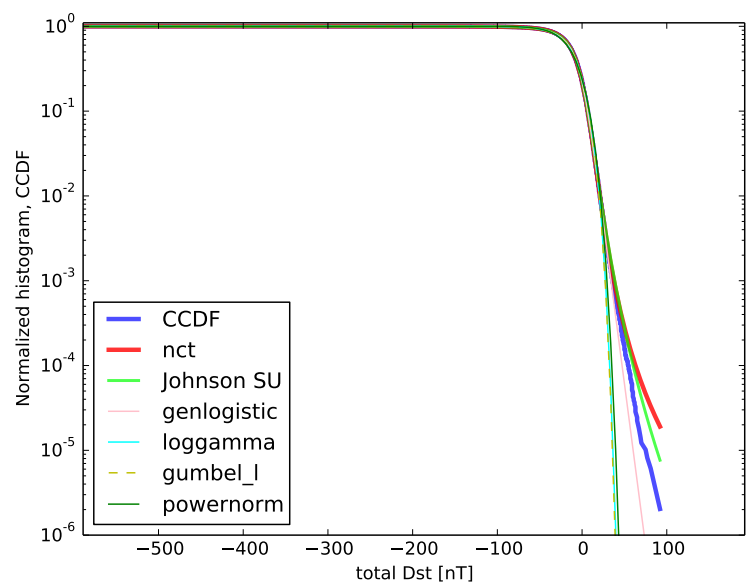

Figure 2. Best fit for Dst different distributions. Top left: Best fit distributions for $|n e g(D s t)|, 1997-2012$. Top right: Best fit distributions for Dst, 1997-2012. Bottom left: Best fits for the $|n e g(D s t)|, 1957-2012$. Bottom right: Best fit distributions for Dst, $1957-2012$. 
Nat. Hazards Earth Syst. Sci. Discuss., https://doi.org/10.5194/nhess-2018-92

Manuscript under review for journal Nat. Hazards Earth Syst. Sci.

Discussion started: 12 April 2018

(c) Author(s) 2018. CC BY 4.0 License.

(c) (i)
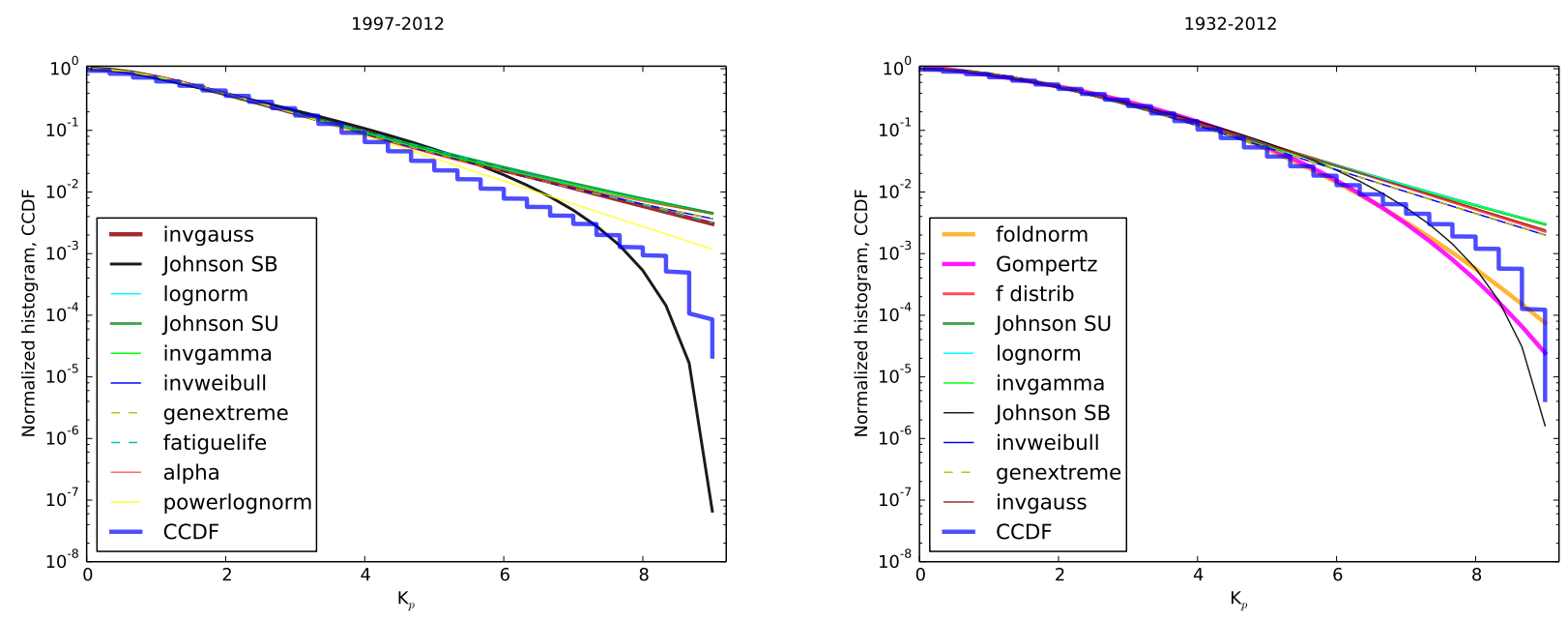

Figure 3. Best fit for $K_{p}$ different distributions. Left: Best fit distributions for $K_{p}, 1997-2012$. Right: Best fit distributions for $K_{p} 1932-2012$.

(Johnson-Su, in pale pink) is about $-175 \mathrm{nT}$. The interval of $n c f$ overlapping again with the CCDF is from -305 to $-365 \mathrm{nT}$. Therefore, with these intersects, we can consider $-70 \mathrm{nT}$ as the threshold for moderate storms, $-175 \mathrm{nT}$ as marking intense storms, and the middle point, approx. as $-330 \mathrm{nT}$, as extreme geomagnetic storm.

For the period 1997-2012, the whole SYM-H is plotted in the right panel of Fig. 1. The best fits are for non-central Student's $5 \quad t$ ('nct') and Johnson-Su.

The best fit distribution functions and Dst CCDF are plotted in Fig. 2. For the negative part of Dst in the period 1997-2012, whose corresponding panel is the upper left, most distributions start departing from the CCDF at around $-75 \mathrm{nT}$. The best fit distribution is the non-central $f$-distribution ( $n c f$, dark grey thick line). In this case, it starts departing around $-90 \mathrm{nT}$ and it intersects again on $-405 \mathrm{nT}$. Some other distributions start departing in a previous point, as around $-38 \mathrm{nT}$ for the inverse gamma (invgamma, grey dashed line), and the generalized extreme value function (GEV, here named 'genextreme', olive dashed line); and $-45 \mathrm{nT}$ for the non-central Student's $t$ ( $n c t$, blue thin line). Other second intercept is seen on the Johnson-Su (pale pink thick line) and lognormal (green lime thick line) at $-395 \mathrm{nT}$.

The negative part of the Dst from 1957 to 2012 is plotted in panel lower left of Fig. 2. The CCDF is best fitted by the noncentral $f$-distribution ( $n c f$, in dark grey thick line). Most functions start departing at $-80 \mathrm{nT}$, but some departs in a previous point as $-70 \mathrm{nT}$ for the non-central Student's ( $n c t$, blue thin line) and (invgamma, grey dashed line). The CCDF starts departing from the $n c f$ at $-150 \mathrm{nT}$ and then it crosses again on $-330 \mathrm{nT}$.

The whole $D s t$ for that period is best fitted by a non-central Student's $t$ for both short and long intervals (in red thick line), shown in the right panels of Fig. 2.

Last, the $K_{p}$ index for the period 1997-2012 and the corresponding best fit distributions are plotted in Fig. 3, left panel. The intersects of the CCDF with the best fit distribution, the inverse gaussian (invgauss, thick brown line) followed by the Johnson$\mathrm{Sb}$ (in black). The intersect with the best fitting function invgauss yields a value of 4 . In addition to this, the intersect with the 
Nat. Hazards Earth Syst. Sci. Discuss., https://doi.org/10.5194/nhess-2018-92

Manuscript under review for journal Nat. Hazards Earth Syst. Sci.

Discussion started: 12 April 2018

(C) Author(s) 2018. CC BY 4.0 License.
Natural Hazards

and Earth System

Sciences

Discussions

(c) (i)

second best fitting function, Johnson-Sb, yields an intersect in the tail, around $7^{+}$. These two points may define thresholds of quiet/disturbed period, and disturbed/very disturbed geomagnetic conditions.

For the period 1932-2012, the departure point of the $K_{p}$ CCDF and all best fit distributions is $K_{p}$ equal to 5 as shown in Fig. 3, right panel. The best fitting distribution is the folded normal (foldnorm, thick yellow-orange line) and the inverse gamma

5 (Gompertz, thick magenta line). These two best fit distributions start departing from the CCDF on $K_{p}$ around 6 , which may mark a disturbed/very disturbed threshold.

The whole distributions, considering positive and negative values (as Dst, SYM-H) are fitted for the sake of illustrative purposes on asymmetric distributions and shown on the corresponding right panels, but thresholds in these cases are not sought out.

We have to take into account that $K_{p}$ is a discrete variable. Therefore we have also tried a set of discrete distributions, as the Poisson, geometric and Planck, shown in Fig. 4. These distributions are minimized through a non-binning dependent log likelihood method. Bins are normalized on density, to match the probability density function (PDF) on every bin. The PDF and the distribution functions yields an intersect of $5^{-}$for the period 1997-2012 and equal to 5 on the period 1932-2012. These trends are similar to the continuous distribution functions, that also gets a value of $K_{p}=5$, which is coincidental to the NOAA scale G1.

1997-2012

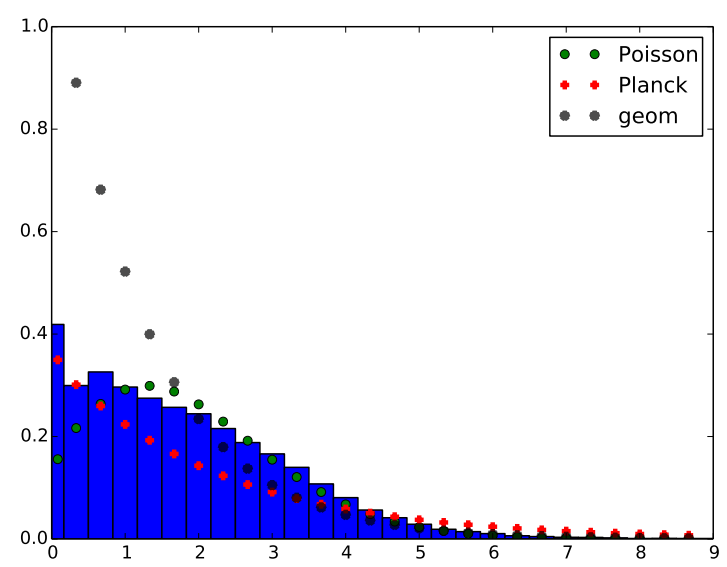

1932-2012

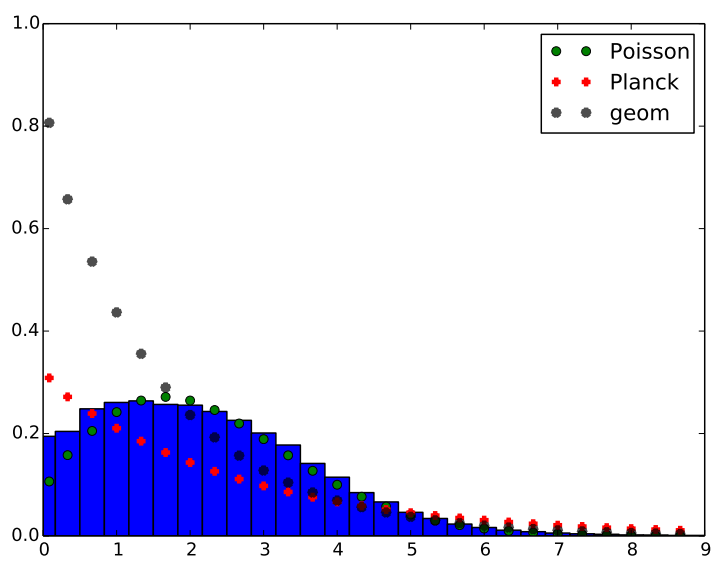

Figure 4. $K_{p}$ different discrete distributions fits. Left: fitted distributions for $K_{p}, 1997-2012$. Right: fitted distributions for $K_{p} 1932-2012$.

\section{Discussion}

An amount of research about distributions applied on different data has been done, such as Dst mainly but also on SYM-H, polar indices, etc. Historically, one of the first applied distributions which may fit the very skewed distribution of geomagnetic disturbances is the lognormal. 
Nat. Hazards Earth Syst. Sci. Discuss., https://doi.org/10.5194/nhess-2018-92

Manuscript under review for journal Nat. Hazards Earth Syst. Sci.

Discussion started: 12 April 2018

(c) Author(s) 2018. CC BY 4.0 License.

Lognormal distribution shapes of the Dst have been commented in Campbell (1996), somehow relating the Dst shape profile (that resembles a lognormal function) to a lognormal distribution. In Aguado et al. (2010), the recovery phase is fitted as a hyperbolic function. However, the disturbance profile shapes will not be further discussed here.

Importantly, also quasi-logarithmic scales are used for defining classes, as for the $K$ index (Mayaud, 1980). In Burlaga

5 and Lazarus (2000), distributions of solar wind are fitted to lognormal or double-peaked lognormal, since two solar wind distributions are employed, for slow and fast solar wind. Pulkkinen et al. (2012) use shifted lognormal for the geoelectric field amplitudes for past events. Love et al. (2016) employ 1-min horizontal component data from several observatories, fitting to threshold-truncated log-normal with least-squares and maximum likelihood method. Also Love et al. (2015) test both (truncated) lognormal and powerlaw Dst distributions, finding that different statistical tests favour the lognormal. Log-normal and powerlaw distributions are related, since some small changes in generative processes which show log-normal distributions may lead to power-law generative processes (Mitzenmacher, 2003). The lognormal in SYM-H and Dst usually scores best in shorter series, and worse for longer series. It also appears for the $K_{p}$ shorter and longer series.

Power-law distributions are extensively used in many fields, mainly because they are scale invariant, e.g. in Newman (2005). A number of power laws appears in X-ray flare energy distribution, solar energetic particle event fluence distribution, auroral activity and radiation belts, due to the consideration of these physical systems as self-critically organized (e.g., Crosby, 2011). For solar flares, a number of power laws are obtained for flare length, volume, time and diffusivity, revealing the fractal nature and the intrinsic relationship to self-organized criticality (SOC) (Aschwanden et al., 2013). In Yermolaev et al. (2013) the relationship between a variety of interplanetary features and Dst values is studied, fitting log-log quadratic functions, employing power law and square-law fits. Power laws are also utilized for relating the geomagnetic disturbance and its rate of change in scatterplots (Tóth et al., 2014).

Power laws appear as the most utilized distribution in Riley (2012) for CME speeds, X-ray flares and geomagnetic events. The CME speed distribution also follows a power law with index equal -3.2. Kataoka (2013) fit power laws $d H$ amplitude distribution, finding $\alpha$ around -3.2 (solar cycle 23) to -3.8 (solar cycle 16); this range is in agreement with the results obtained here. Wanliss and Weygand (2007) highlight the lack of characteristic time scale for power laws employed on storm waiting time with $S Y M-H$ index and other interplanetary medium parameters. They obtain an index slope of -1.2 using a powerlaw with an exponential cutoff.

Power laws are also the most likely function for power spectra of a myriad of physical magnitudes: for solar wind characteristics, in Burlaga and Lazarus (2000) the power spectra of the solar wind is analysed, finding that the power spectral index for interplanetary medium density is $\alpha=-5 / 3$ (coincidental with that of Kolmogorov turbulence index, and also coincidental with the fractral powerlaw for 3D events and volumes (Aschwanden et al., 2013). For proton temperature $\alpha$ also gets values in this range. The $\alpha$ parameter between -1 and -2 that they obtained may indicate small-scale structures in the solar wind fluctuations. In Zimbardo et al. (2008, and references therein) the power spectra index of the magnetic turbulence spectrum $\left(\mathrm{B}^{2}\right.$ vs. frequency) in the magnetosphere is investigated; more specifically, in the magnetosheath goes from $-7 / 3$ (implying turbulent cascade) to -2.6. Kiyani et al. (2015) analyse the different regimes in magnetic field fluctuations. In Burlaga and 
Nat. Hazards Earth Syst. Sci. Discuss., https://doi.org/10.5194/nhess-2018-92

Manuscript under review for journal Nat. Hazards Earth Syst. Sci.

Discussion started: 12 April 2018

(c) Author(s) 2018. CC BY 4.0 License.
Natural Hazards

and Earth System

Sciences

Discussions

poor score for $K_{p}$ and $D s t$. They are absent in $S Y M-H$.

Next interesting distribution family is the Generalized Extreme Value distributions (GEV). The 'heavy-tailed distributions' as GEV distributions prove useful for taking into account the very extreme and unlikely events. The branch of GEV distributions (Fréchet, Gumbell or Weibull, depending whether the shape factor $c$ is larger than, equal to, or less than zero) is widely used which means that they are not the best possible fit function.

In Tsubouchi and Omura (2007), the GEV distribution (a Gumbel distribution) is used in Dst for estimating the mean excess function, and the waiting time is estimated through a Poisson distribution (as made also by e.g. Kataoka (2013); Love (2012)). They also estimate an extreme value threshold of $-280 \mathrm{nT}$ by the different distribution regimes using a powerlaw index of -4.9 for the extreme-event tail. Nikitina et al. (2016) also employ GEV distribution for subauroral, auroral and polar geomagnetic data aiming at return period estimation. GEV distribution are also used for statistical distribution fit of flares in Curto et al. (2016). Thomson et al. (2011) use GEV in addition to a Generalized Pareto distribution.Wintoft et al. (2016) also apply GEV for the analyses of the return period on the derivative of the magnetic field and modelled electric field. Here we fit, for all indices, some distributions from this family, as obviously the 'genextreme' (proper GEV), and/or Frechet $L$ (left skewed), which is the Weibull maximum; Frechet $R$ (right skewed) is equal to the Weibull minimum. Gumbel $R$ is the right skewed Gumbel distribution. These distributions appear for Dst and SYM-H, but with relatively large $D$.

All these different works are dependent on data sample sizes, how evenly these data are distributed, and most importantly, the fact that they are data from a variety of geomagnetic indices, sometimes as single-observatory sets, and sometimes as an average of different magnetometer data; therefore distributions applied may be different. The nature of the data values is also important: usually the geomagnetic components that are averaged on different observatories may exhibit smaller values than single-observatory data; the values would be even higher when single high-latitude observatories are considered.

It is also relevant to consider the length of the sequence: $S Y M-H$ consists of more than 8 million datapoints, comprising more than 15 years due to its 1-min cadence. To form an equivalent dataset Dst series of that size - or another 1-h index -, a 960-year series would be necessary, or 2880 years of $3-\mathrm{h} K_{p}$ or equivalent in cadence. The series employed here are considered a large number set in statistics. We show that $D$ exhibits lower values (better fit) on the longest series of each index.

Very interestingly, trends are kept for $S Y M-H$ and $D s t$. We observe that the negative values, corresponding to storm values, always fit best as a non-central $f$, which is a non-center $f$ distribution, while $f$ is related to beta distribution; the whole distribution (both positive and negative) is best fit to the non-central Student's $t$, which is the generalized $t$ distribution, also related to beta.

With less data (or maybe less strong geomagnetic storms), distributions usually can exhibit a good fit to Johnson-Sb bounded-, but when more data (or maybe more extreme values) are added, distributions tend to be Johnson-Su, unbounded. For the whole Dst and SYM-H, the hyperbolic-sine Johnson-Su has similarity in shape compared to Student's $t-$ and its non-central version $n c t-$, which is also a generalized hyperbolic distribution. 
Nat. Hazards Earth Syst. Sci. Discuss., https://doi.org/10.5194/nhess-2018-92

Manuscript under review for journal Nat. Hazards Earth Syst. Sci.

Discussion started: 12 April 2018

(c) Author(s) 2018. CC BY 4.0 License.
Natural Hazards

and Earth System

Sciences

Discussions

\section{(c) (i)}

$K_{p}$ distribution functions best fits differ from those applied on the continuous indices. For this index, the inverse gaussian (invgauss, related to the gamma function), the Johnson-Sb, folded normal (foldnorm), Gompertz (which is not that closely related to gamma) are the best fits. As expected, $D$ is higher than the continuous indices, since its fitting cannot be that good. For the $K_{p}$ shorter series, Johnson-Sb scores better than Johnson-Su, since data are more limited on density; and the opposite happens for the longer series. In any case, the bounds are on 0 and 9.

5 Some of the best fit presented here are beta functions, which are closely linked to the gamma function. Love (2012) uses discrete Poisson distributions that are transformed into a gamma functions for frequentist and bayesian modelling.

Both $f, t$ - and power-law distributions are unbounded, in addition to the Johnson-Su (hyperbolic-sine type), among others. This means that, statistically, the tails are not limited. Therefore, the best fit functions for Dst and $S Y M-H$ do not have upper limits, theoretically. This is in agreement with the results in Wintoft et al. (2016). However, the physical parameters that trigger geomagnetic storms may be upper limited by the finiteness on impulsivity of solar features, such as CME speed, solar wind speed, shocks, which cannot take every kind of values, but have an empirical upper limit (finite size effect, e.g. Newman, 2005).

In addition to these considerations, thresholds are used in a reverse mode in this paper: first we fit data to a distribution and then we obtain thresholds. The usual reasoning, which is the utilization of ad-hoc thresholds for calculations, appear, e.g., for mean residual life computation to estimate return periods, e.g., in Thomson et al. (2011), or as percentile comparison in Nikitina et al. (2016) in addition to the use of Q-Q plots (diagnostics tool based on the quartile comparison of a chosen distribution and the data intended to be fitted), helping in the task of checking the goodness-of-fit of statistical distribution. Kataoka and Ngwira (2016) set some alert thresholds for geomagnetic disturbance and its rate of change, corresponding to different geomagnetic transients, without statistical distribution fit; or the percentile choice for proving the threshold independence of power laws in Wanliss and Weygand (2007).

As we mention before, no time between geomagnetic disturbances is considered. As future work, this can be considered for clustering proper geomagnetic storms; then, block-maxima related distributions to those obtained here would be expected.

\section{Conclusions}

1. For the continuous indices which exhibit positive and negative values, these negative values usually follow the noncentral $f$ distribution, while the whole distributions best fit is non-central $t$. Best fits (lower $D$ ) appear always in the largest datasets.

2. For the index $K_{p}$, we get two intersects in the period 1997-2012, which are $K_{p}$ equal to 4 and $7^{+}$. For the $1932-$ 2012 period, the obtained departing point is $K_{p}=5$, in agreement with the minimum threshold for geomagnetic storms defined by the NOAA $G$ scale as G1 ("minor storm"). Data and the best fitting function yield an intersect of $K_{p}=6$, as NOAA $G$ scale as G2 ("moderate storm"), for the longer series. These two thresholds may mark the difference between quietness/storm time, and disturbed/very disturbed conditions. Additionally, the discrete distribution yields a value of $K_{p}$ about 5 for both short and long series. 
Nat. Hazards Earth Syst. Sci. Discuss., https://doi.org/10.5194/nhess-2018-92

Manuscript under review for journal Nat. Hazards Earth Syst. Sci.

Discussion started: 12 April 2018

(c) Author(s) 2018. CC BY 4.0 License.

(c) (i)

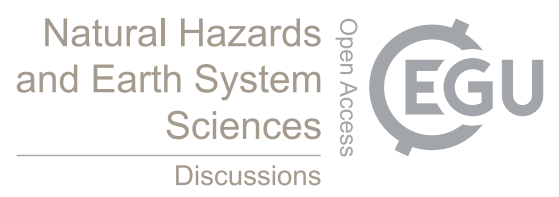

3. For the Dst index, the intersect points for the period 1957-2012 is about $-80 \mathrm{nT},-150 \mathrm{nT}$ and $-330 \mathrm{nT}$, which can define the thresholds for moderate, intense and extreme geomagnetic storms. For the shorter series, the intersect is on $-90 \mathrm{nT}$ and $-405 \mathrm{nT}$.

4. For the $S Y M-H$ index, the intersect points mark thresholds of about $-70 \mathrm{nT},-175$ and $-335 \mathrm{nT}$, that may define the categories mentioned above.

$5 \quad$ 5. These thresholds can be useful for geomagnetic activity dependent users, including GIC-aware users.

6. This method is applicable to any kind of geomagnetic index, for example to other global or regional ones.

Competing interests. We authors declare that they do not have competing interests.

Acknowledgements. We thank INTERMAGNET for promoting high standards of magnetic observatory practice www.intermagnet.org.

We acknowledge the use of Python and their statistical packages for programming.

We thank funding from MINECO project AYA2016-80881-P (including AEI/FEDER funds, EU). 
Nat. Hazards Earth Syst. Sci. Discuss., https://doi.org/10.5194/nhess-2018-92

Manuscript under review for journal Nat. Hazards Earth Syst. Sci.

Discussion started: 12 April 2018

(C) Author(s) 2018. CC BY 4.0 License.

\section{References}

Aguado, J., Cid, C., Saiz, E., and Cerrato, Y.: Hyperbolic decay of the Dst Index during the recovery phase of intense geomagnetic storms, Journal of Geophysical Research (Space Physics), 115, A07220, https://doi.org/10.1029/2009JA014658, 2010.

Alstott, J., Bullmore, E., and Plenz, D.: powerlaw: A Python Package for Analysis of Heavy-Tailed Distributions, PLoS ONE, 9, e85 777, https://doi.org/10.1371/journal.pone.0085777, 2014.

5 Aschwanden, M. J., Zhang, J., and Liu, K.: Multi-wavelength Observations of the Spatio-temporal Evolution of Solar Flares with AIA/SDO.

I. Universal Scaling Laws of Space and Time Parameters, Astrophysical Journal, 775, 23, https://doi.org/10.1088/0004-637X/775/1/23, 2013.

Bartels, J. and Veldkamp, J.: International data on magnetic disturbances, first quarter, 1949, Journal of Geophysical Research, 54, 295-299, https://doi.org/10.1029/JZ054i003p00295, http://dx.doi.org/10.1029/JZ054i003p00295, 1949.

Bartels, J., Heck, N. H., and Johnston, H. F.: The three-hour-range index measuring geomagnetic activity, Terrestrial Magnetism and Atmospheric Electricity (Journal of Geophysical Research), 44, 411, https://doi.org/10.1029/TE044i004p00411, 1939.

Burlaga, L. F. and Lazarus, A. J.: Lognormal distributions and spectra of solar wind plasma fluctuations: Wind 1995-1998, Journal of Geophysical Research, 105, 2357-2364, https://doi.org/10.1029/1999JA900442, 2000.

Campbell, W. H.: Geomagnetic storms, the Dst ring-current myth and lognormal distributions, Journal of Atmospheric and Terrestrial Physics, 58, 1171-1187, 1996.

Cid, C., Saiz, E., Guerrero, A., Palacios, J., and Cerrato, Y.: A Carrington-like geomagnetic storm observed in the 21st century, Journal of Space Weather and Space Climate, 5, A16, https://doi.org/10.1051/swsc/2015017, 2015.

Cid, C., Palacios, J., Guerrero, A., Saiz, E., and Cerrato, Y.: Efficient space weather services: a critical issue for future NATO operations, in: Considerations for Space and Space-Enabled Capabilities in NATO Coalition Operations, STO-MP-SCI-283, 2016.

Crosby, N. B.: Frequency distributions: from the sun to the earth, Nonlinear Processes in Geophysics, 18, 791-805, https://doi.org/10.5194/npg-18-791-2011, 2011.

Curto, J. J., Castell, J., and Del Moral, F.: Sfe: waiting for the big one, Journal of Space Weather and Space Climate, 6, A23, https://doi.org/10.1051/swsc/2016018, 2016.

Eastwood, J. P., Biffis, E., Hapgood, M. A., Green, L., Bisi, M. M., Bentley, R. D., Wicks, R., McKinnell, L.-A., Gibbs, M., and Burnett, C.: The Economic Impact of Space Weather: Where Do We Stand?, Risk Analysis, 37, 206-218, https://doi.org/10.1111/risa.12765, http://dx.doi.org/10.1111/risa.12765, 2017.

Echer, E., Gonzalez, W. D., and Tsurutani, B. T.: Interplanetary conditions leading to superintense geomagnetic storms $($ Dst $<=-250 \mathrm{nT})$ during solar cycle 23, Geophysical Research Letters, 35, L06S03, https://doi.org/10.1029/2007GL031755, $2008 \mathrm{a}$.

Echer, E., Gonzalez, W. D., Tsurutani, B. T., and Gonzalez, A. L. C.: Interplanetary conditions causing intense geomagnetic storms (Dst $<=-100 \mathrm{nT}$ ) during solar cycle 23 (1996-2006), Journal of Geophysical Research (Space Physics), 113, A05221, https://doi.org/10.1029/2007JA012744, 2008b.

Gonzalez, W. D., Joselyn, J. A., Kamide, Y., Kroehl, H. W., Rostoker, G., Tsurutani, B. T., and Vasyliunas, V. M.: What is a geomagnetic storm?, Journal of Geophysical Research, 99, 5771-5792, https://doi.org/10.1029/93JA02867, 1994.

Gonzalez, W. D., Echer, E., Clúa de Gonzalez, A. L., Tsurutani, B. T., and Lakhina, G. S.: Extreme geomagnetic storms, recent Gleissberg cycles and space era-superintense storms, Journal of Atmospheric and Solar-Terrestrial Physics, 73, 1447-1453, https://doi.org/10.1016/j.jastp.2010.07.023, 2011. 
Nat. Hazards Earth Syst. Sci. Discuss., https://doi.org/10.5194/nhess-2018-92

Manuscript under review for journal Nat. Hazards Earth Syst. Sci.

Discussion started: 12 April 2018

(c) Author(s) 2018. CC BY 4.0 License.

Grafe, A.: Are our ideas about Dst correct?, Annales Geophysicae, 17, 1-10, https://doi.org/10.1007/s00585-999-0001-0, http://dx.doi.org/ 10.1007/s00585-999-0001-0, 1998.

Guerrero, A., Cid, C., Saiz, E., Palacios, J., and Cerrato, Y.: Dispositivo y procedimiento de obtención de la perturbación geomagnética local a latitudes medias. Spanish Patents, Pub. No: WO/2017/174843, 2016.

Karinen, A. and Mursula, K.: Correcting the Dst index: Consequences for absolute level and correlations, Journal of Geophysical Research: Space Physics, 111, n/a-n/a, https://doi.org/10.1029/2005JA011299, http://dx.doi.org/10.1029/2005JA011299, a08207, 2006.

Kataoka, R.: Probability of occurrence of extreme magnetic storms, Space Weather, 11, 214-218, https://doi.org/10.1002/swe.20044, 2013.

Kataoka, R. and Ngwira, C.: Extreme geomagnetically induced currents, Progress in Earth and Planetary Science, 3, 23, https://doi.org/10.1186/s40645-016-0101-x, 2016.

Kiyani, K. H., Osman, K. T., and Chapman, S. C.: Dissipation and heating in solar wind turbulence: from the macro to the micro and back again, Philosophical Transactions of the Royal Society of London A: Mathematical, Physical and Engineering Sciences, 373, https://doi.org/10.1098/rsta.2014.0155, 2015.

Love, J. J.: Credible occurrence probabilities for extreme geophysical events: Earthquakes, volcanic eruptions, magnetic storms, Geophysical Research Letters, 39, L10301, https://doi.org/10.1029/2012GL051431, 2012.

Love, J. J., Rigler, E. J., Pulkkinen, A., and Riley, P.: On the lognormality of historical magnetic storm intensity statistics: Implications for extreme-event probabilities, Geophysical Research Letters, 42, 6544-6553, https://doi.org/10.1002/2015GL064842, 2015.

Love, J. J., Coïsson, P., and Pulkkinen, A.: Global statistical maps of extreme-event magnetic observatory 1 min first differences in horizontal intensity, Geophysical Research Letters, 43, 4126-4135, https://doi.org/10.1002/2016GL068664, 2016.

Mayaud, P. N.: Derivation, Meaning, and Use of Geomagnetic Indices, Washington DC American Geophysical Union Geophysical Monograph Series, 22, 607, https://doi.org/10.1029/GM022, 1980.

Mitzenmacher, M.: A Brief History of Generative Models for Power Law and Lognormal Distributions, INTERNET MATHEMATICS, 1, 226-251, 2003.

Newman, M.: Power laws, Pareto distributions and Zipf's law, Contemporary Physics, 46, 323-351, https://doi.org/10.1080/00107510500052444, 2005.

Ngwira, C. M., Pulkkinen, A. A., Bernabeu, E., Eichner, J., Viljanen, A., and Crowley, G.: Characteristics of extreme geoelectric fields and their possible causes: Localized peak enhancements, Geophysical Research Letters, 42, 6916-6921, https://doi.org/10.1002/2015GL065061, http://dx.doi.org/10.1002/2015GL065061, 2015GL065061, 2015.

Nikitina, L., Trichtchenko, L., and Boteler, D. H.: Assessment of extreme values in geomagnetic and geoelectric field variations for Canada, Space Weather, 14, 481-494, https://doi.org/10.1002/2016SW001386, 2016.

Palacios, J., Cid, C., Guerrero, A., Saiz, E., Cerrato, Y., Rodríguez-Bouza, M., Rodríguez-Bilbao, I., Herraiz, M., and Rodríguez-Caderot, G.: The Spanish Space Weather Service SeNMEs. A Case Study on the Sun-Earth Chain, in: Coimbra Solar Physics Meeting: Ground-based Solar Observations in the Space Instrumentation Era, edited by Dorotovic, I., Fischer, C. E., and Temmer, M., vol. 504 of Astronomical Society of the Pacific Conference Series, p. 131, 2016.

Pulkkinen, A., Bernabeu, E., Eichner, J., Beggan, C., and Thomson, A. W. P.: Generation of 100-year geomagnetically induced current scenarios, Space Weather, 10, 04003, https://doi.org/10.1029/2011SW000750, 2012.

Riley, P.: On the probability of occurrence of extreme space weather events, Space Weather, 10, 02012, https://doi.org/10.1029/2011SW000734, 2012. 
Nat. Hazards Earth Syst. Sci. Discuss., https://doi.org/10.5194/nhess-2018-92

Manuscript under review for journal Nat. Hazards Earth Syst. Sci.

Discussion started: 12 April 2018

(c) Author(s) 2018. CC BY 4.0 License.

(c) (i)

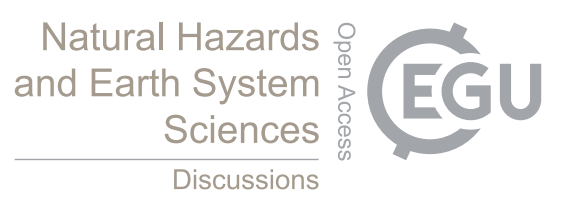

Rostoker, G.: Geomagnetic indices., Reviews of Geophysics and Space Physics, 10, 935-950, https://doi.org/10.1029/RG010i004p00935, 1972.

Saiz, E., Guerrero, A., Cid, C., Palacios, J., and Cerrato, Y.: Searching for Carrington-like events and their signatures and triggers, Journal of Space Weather and Space Climate, 6, A6, https://doi.org/10.1051/swsc/2016001, 2016.

Schulte in den Bäumen, H., Moran, D., Lenzen, M., Cairns, I., and Steenge, A.: How severe space weather can disrupt global supply chains, Natural Hazards and Earth System Sciences, 14, 2749-2759, https://doi.org/10.5194/nhess-14-2749-2014, https://www. nat-hazards-earth-syst-sci.net/14/2749/2014/, 2014.

Sugiura, M. and Kamei, T.: Equatorial Dst index 1957-1986, in: IAGA Bulletin, edited by by A. Berthelier, E. and Menvielle, M., vol. 40 of International Union of Geodesy and Geophysics. Association of Geomagnetism and Aeronomy, http://wdc.kugi.kyoto-u.ac.jp/dstdir/dst2/ onDstindex.html, 1991.

10 Thomson, A. W. P., Dawson, E. B., and Reay, S. J.: Quantifying extreme behavior in geomagnetic activity, Space Weather, 9, S10001, https://doi.org/10.1002/2011SW000696, 2011.

Tóth, G., Meng, X., Gombosi, T. I., and Rastätter, L.: Predicting the time derivative of local magnetic perturbations, Journal of Geophysical Research (Space Physics), 119, 310-321, https://doi.org/10.1002/2013JA019456, 2014.

Tsubouchi, K. and Omura, Y.: Long-term occurrence probabilities of intense geomagnetic storm events, Space Weather, 5, S12003, https://doi.org/10.1029/2007SW000329, 2007.

Tsurutani, B. T., Gonzalez, W. D., Tang, F., and Lee, Y. T.: Great magnetic storms, Geophysical Research Letters, 19, 73-76, https://doi.org/10.1029/91GL02783, http://dx.doi.org/10.1029/91GL02783, 1992.

Wanliss, J. A. and Weygand, J. M.: Power law burst lifetime distribution of the SYM-H index, Geophysical Research Letters, 34, L04107, https://doi.org/10.1029/2006GL028235, 2007.

Wheatley, S., Sovacool, B., and Sornette, D.: Of Disasters and Dragon Kings: A Statistical Analysis of Nuclear Power Incidents and Accidents, Risk Analysis, 37, 99-115, https://doi.org/10.1111/risa.12587, http://dx.doi.org/10.1111/risa.12587, 2017.

Wilks, D. S., ed.: Statistical Methods in the Atmospheric Sciences, vol. 100 of International Geophysics, Academic Press, https://doi.org/https://doi.org/10.1016/B978-0-12-385022-5.00022-1, 2011.

Wintoft, P., Viljanen, A., and Wik, M.: Extreme value analysis of the time derivative of the horizontal magnetic field and computed electric field, Annales Geophysicae, 34, 485-491, https://doi.org/10.5194/angeo-34-485-2016, https://www.ann-geophys.net/34/485/2016/, 2016.

Yermolaev, Y. I., Lodkina, I. G., Nikolaeva, N. S., and Yermolaev, M. Y.: Occurrence rate of extreme magnetic storms, Journal of Geophysical Research (Space Physics), 118, 4760-4765, https://doi.org/10.1002/jgra.50467, 2013.

Zimbardo, G., Greco, A., Veltri, P., Voros, Z., and Taktakishvili, A. L.: Magnetic turbulence in and around the Earth's magnetosphere, Astrophysics and Space Sciences Transactions, 4, 35-40, https://doi.org/10.5194/astra-4-35-2008, 2008. 